\title{
BIOINSPIRED DEVICES
}





\section{BIOINSPIRED DEVICES}

Emulating Nature's Assembly and Repair Process

Eugene C. Goldfield

Harvard University Press

Cambridge, Massachusetts

London, England

2018 
Copyright (c) 2018 by the President and Fellows of Harvard College All rights reserved

Printed in the United States of America

First printing

Library of Congress Cataloging-in-Publication Data

Names: Goldfield, Eugene Curtis, author.

Title: Bioinspired devices : emulating naturés assembly and repair process / Eugene C. Goldfield.

Description: Cambridge, Massachusetts : Harvard University Press, 2018. |

Includes bibliographical references and index.

Identifiers: LCCN 2017009706 | ISBN 9780674967946 (alk. paper)

Subjects: LCSH: Biomedical engineering. | Biomimicry. | Medical innovations.

Classification: LCC R856 .G66 2018 | DDC 610.28-dc23

LC record available at https://lccn.loc.gov/2017009706

Cover art: (top) Controlled synthesis of hierarchical complex structures. False-colored SEMs showing a field of purple $\mathrm{SrCO} 3-\mathrm{SiO} 2$ vases containing $\mathrm{SrCO} 3-\mathrm{SiO} 2$ stems (green) that were subsequently opened with a CO2 pulse (blue). Image courtesy of Wim L. Noorduin. (bottom) Microscopic view of nanotechnology, 3D rendering (gecko753/ Getty Images).

Cover design by Jill Breitbarth 
For Beverly 
